\title{
Radiation-Induced Myopathy Developing in a Hodgkin Lymphoma Patient: An Autopsy Case with Systemic Muscle Sampling
}

\author{
Jun Ando ${ }^{a}$ Yuji Ueno ${ }^{b}$ Hajime Yasuda $^{a}$ Miki Ando $^{a}$ Yoko Edahiro $^{a}$ \\ Tadahiro Honda $^{a}$ Masashi Takanashi ${ }^{b}$ Daisuke Taniguchi ${ }^{b}$

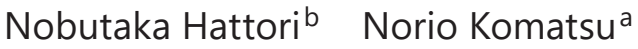 \\ aDepartment of Hematology, Juntendo University School of Medicine, Tokyo, Japan; \\ bepartment of Neurology, Juntendo University School of Medicine, Tokyo, Japan
}

\section{Keywords}

Radiation-induced myopathy · Radiotherapy · Myopathy · Hodgkin's lymphoma

\begin{abstract}
Radiation-induced myopathy (RIM) is a rare complication occurring years after radiotherapy. RIM basically occurs within the irradiation field, but some cases have been reported to be accommodated by myopathy outside the irradiation field, and the actual extent of RIM is obscure. The presented case also showed decreased MMT scores and abnormal needle electromyography results in the muscles outside the irradiated field, and the patient was initially thought to have RIM both within and outside the irradiated field. However, while systemic postmortem muscle sampling revealed prominent myopathy in line with RIM in the irradiated muscles, only mild myogenic changes that could be explained by other causes such as agerelated sarcopenia, radiculopathy, and disuse atrophy were observed in the non-irradiated muscles. The number of biopsy sites in live patients is limited due to the invasive nature of the procedure, but we were privileged to systemically evaluate the extent of myopathy through multiple muscle sampling including muscles both inside and outside of the irradiation field because this was an autopsy case. Through the presented case, we conclude that RIM is a phenomenon most probably limited to the muscles within the irradiated field, and myopathy outside the irradiation is due to other causes.




\section{Introduction}

Prognosis of Hodgkin's lymphoma has improved dramatically, and in such an era, late complications associated with radiation therapy have become one of the biggest concerns. Secondary malignancies and cardiovascular diseases are major late complications, while neuromuscular complications such as radiation-induced myopathy (RIM) are rare [1]. RIM not only drastically impairs quality of life, but also can be life threatening because of subsequent respiratory failure. RIM has been reported to occasionally involve muscles outside the irradiation field, but the actual extent of involvement is obscure [2,3]. We report an autopsy case of a patient with RIM, in which we were able to systemically evaluate the extent of muscle atrophy through multiple and systemic muscle sampling including muscles both inside and outside of the irradiation field.

\section{Case Report}

A 79-year-old female with a history of mixed cellularity classic Hodgkin lymphoma treated with mantle field radiotherapy ( $40 \mathrm{~Gy}$ ) 19 years ago complained of progressive muscle weakness of the neck and shoulders appearing in May 2017. On initial examination in September 2017, the patient presented with dysphagia but not dysarthria. Muscle strengths were 5 in both upper limbs, trapezius muscles and neck extensors and flexors, 4 in both proximal lower limbs, and 5 in both distal lower limbs on Modified Medical Research Council's manual muscle testing (MMT) scores. The patient presented with head drop together with contracture of bilateral shoulder joints (Fig. 1A). There was no camptocormia. State of consciousness, cranial nerves, deep tendon reflexes, sensation, and autonomic functions were normal. Serum creatinine kinase (CK) level was elevated (434 U/L, normal limits 47-200 U/L). Anti-aminoacyl tRNA synthetase (ARS) and anti-histidyl transfer RNA synthetase (Jo) antibodies were negative. Recurrence of Hodgkin's lymphoma and development of other malignancies were not detected. MRI of the shoulders revealed symmetrical atrophy of the
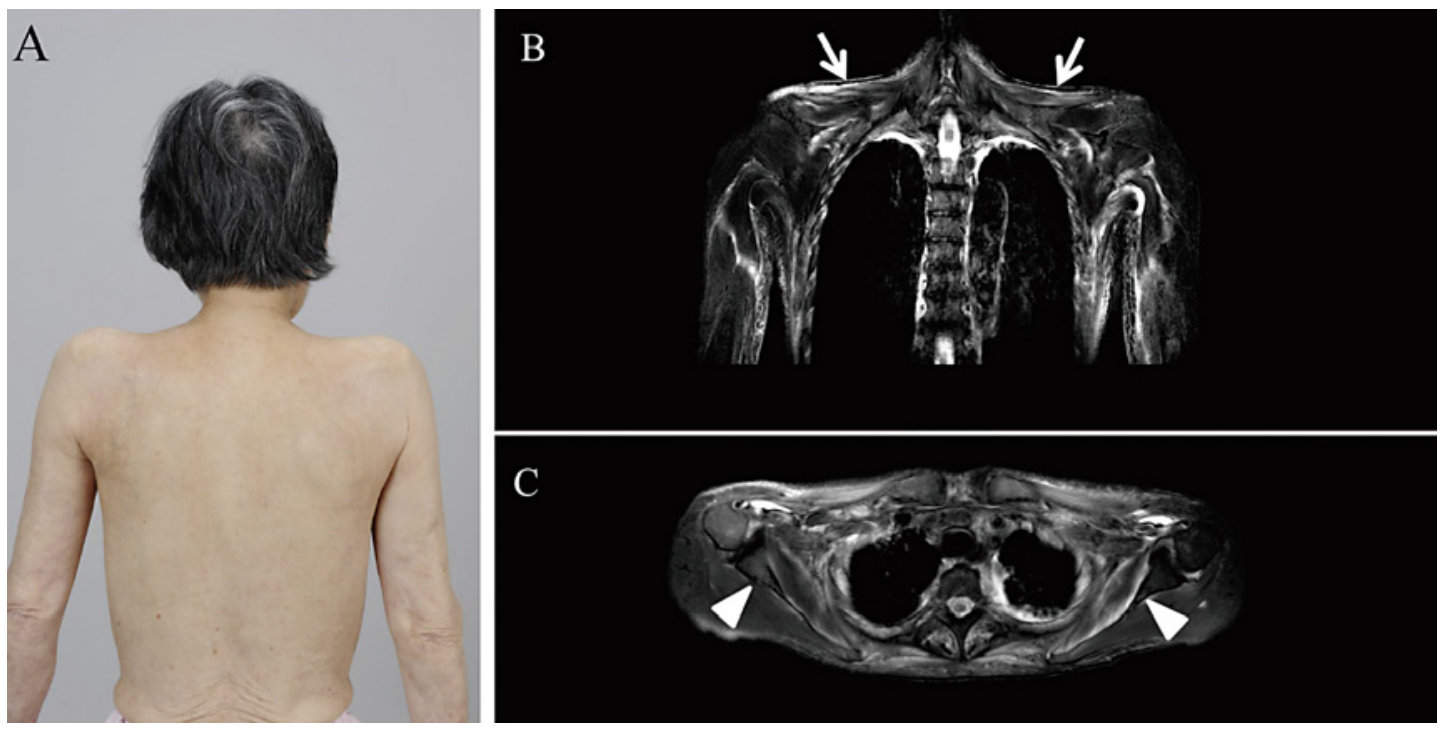

Fig. 1. Posterior view showing marked muscle atrophy of the shoulders (A), and T2-weighted MRI showing atrophies of trapezius (B) and subscapularis (C) muscles. 


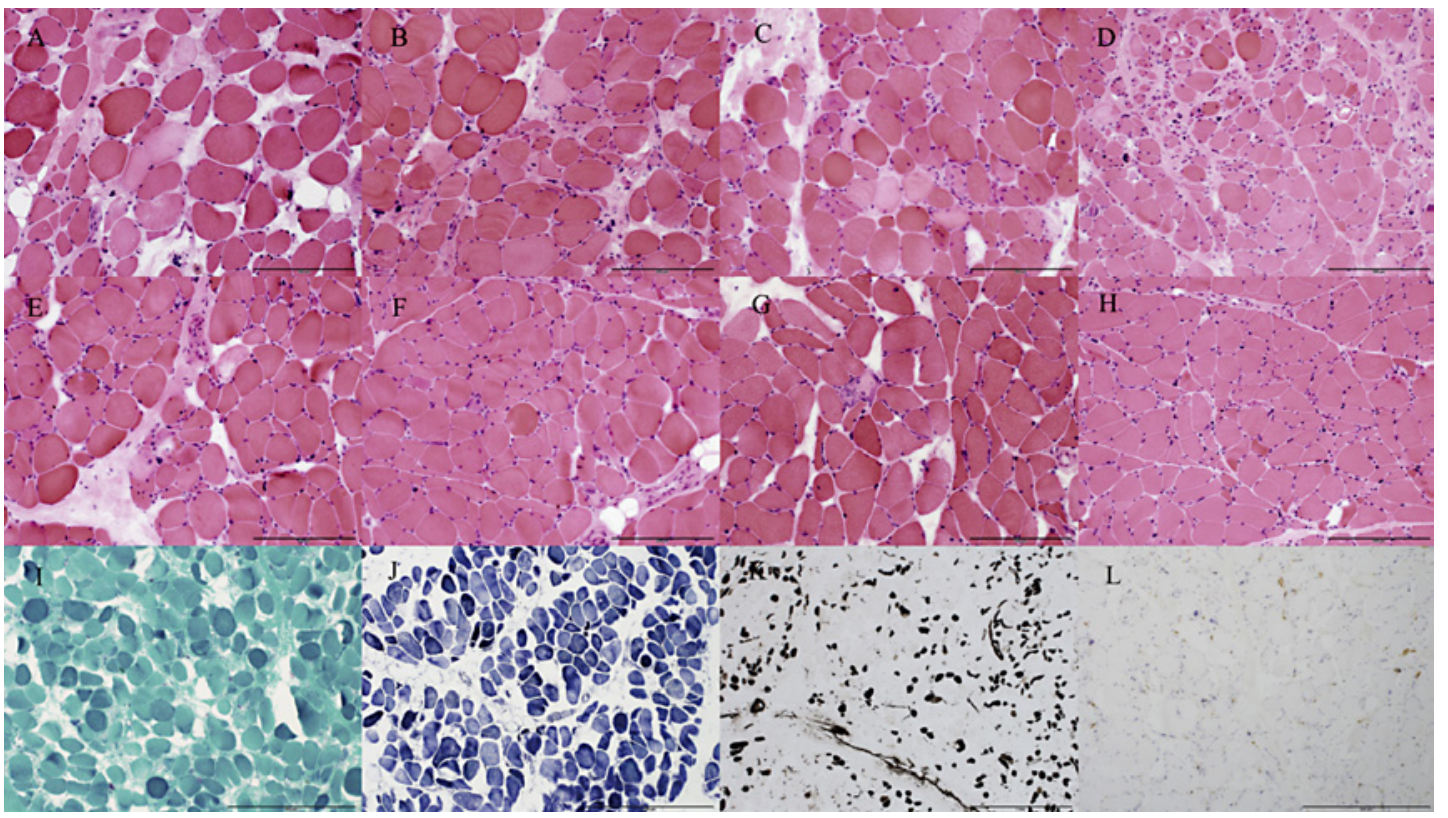

Fig. 2. Autopsy muscle pathology with H\&E staining of trapezius (A), sternocleidomastoid (B), deltoid (C), intercostal (D), biceps brachii (E), brachioradialis (F), dorsal interosseous (G), and vastus lateralis (H) muscles showing myogenic changes such as round-shaped, various sized, necrotic and regenerative fibers, and neurogenic changes such as small angulated fibers and pyknotic nuclear clumps. The fibers of directly irradiated muscles (A-D) showed marked degenerative changes, whereas non-irradiated and remote muscles (E-H) showed no or modest changes. Gomori-trichrome stain revealed no nemaline bodies (I). Type 1 muscle fibers were dominant upon nicotinamide adenine dinucleotide tetrazolium reductase ( $\mathbf{J}$ ) and routine ATPase (pH 9.4) (K) staining. Inflammatory infiltration of lymphocytes in the muscle tissue was not detected upon leukocyte common antigen staining (L).

subscapularis and trapezius muscles (Fig. 1B, C). Nerve conduction studies did not demonstrate any specific findings related to her symptoms, but needle electromyography showed fibrillations in trapezius, 1st dorsal interossei, and thoracic paraspinal muscles and positive sharp waves in trapezius muscles, as well as polyphasic motor unit potentials moderately in trapezius muscles and mildly in 1st dorsal interossei, thoracic paraspinal, masseter, and vastus lateralis muscles. Two months after initial examination, she developed progressive dysphagia complicated by severe aspiration pneumonia and died despite heavy treatment with antibiotics. Postmortem pathological studies using frozen muscle tissues revealed myogenic changes including variation in muscle fiber size, admixture of necrotic and regenerating muscle fibers, increase in endomysial connective and fatty tissue, and neurogenic changes such as pyknotic nuclear clumps and small angular fibers. These findings were prominent in the muscles which were directly irradiated (trapezius, sternocleidomastoid, intercostal, and deltoid muscles), but muscles outside the irradiated field (triceps brachii, brachioradialis, dorsal interosseous, and vastus lateralis muscles) also showed mild myogenic and neurogenic fiber changes (Fig. 2A-L). 
Ando et al.: RIM Developing in a Hodgkin Lymphoma Patient

\section{Discussion}

According to previous studies, the most common presentations of RIM within the irradiated field are head drop, neck pain, and axial and periscapular muscle weakness, but in rare cases non-irradiated muscles have also been reported to be affected in patients with RIM [2-6]. The presented case not only had RIM within the irradiated field, but also showed decreased MMT scores and abnormal needle electromyography results concerning the muscles outside the irradiated field, and the patient was initially thought to have RIM both within and outside the irradiated field. However, while systemic postmortem muscle sampling revealed prominent myopathy in line with RIM in the irradiated muscles, only mild myogenic changes that could be explained by other causes such as age-related sarcopenia and radiculopathy, and disuse atrophy were observed in the non-irradiated muscles. Ghosh and Milone [2] have reported pathologically proven myopathies outside the irradiated field in 2 patients with RIM. However, one of these patients had a family history of hyper CK-emia, and most likely had an inherited myopathy. Therefore, there was only one patient in this report with pathologically proven myopathy outside the irradiated field for which the etiology could have been due to RIM, but the authors concluded that a coexisting myopathy of indeterminate type was most probable. Van Leeuwen-Segarceanu et al. [3] reported a series of patients with RIM, but they did not perform muscle biopsies, and found predominantly neuropathic damage on needle electromyographs in the muscles outside the irradiation field. They speculated that radiation-evoked damage to the nerve roots and brachial plexus were the cause. Thus, despite reports of weakness in muscles outside the irradiated field exist, whether these are the result of RIM remains obscure. A major obstacle is that due to the invasive nature, the number of muscle biopsies possible in live patients is limited. Because ours was an autopsy case, we were privileged to carry out multiple and systemic muscle sampling and pathological analysis in the same individual both within and outside the irradiated field. Despite the large number of sampling sites, evidence supporting RIM in muscles outside the irradiation field could not be found in the presented case, and it is likely that RIM is a phenomenon restricted to the muscles directly irradiated. Previous reports of muscle weakness outside the radiation field may be due to upstream neurologic damage or may simply be secondary to decreased activity of daily life due to true RIM of irradiated muscles.

The prognosis of patients with RIM is dismal, and Ghosh and Milone [2] have reported that 2 out of 21 patients died of neuromuscular respiratory failure, and 6 out of 8 patients undergoing pulmonary function tests showed reduced maximal respiratory pressures. Even when not life-taking, symptoms such as head drop drastically impair quality of life. It has been reported that RIM patients benefit from sport activities and visiting physiotherapists [3]. Therefore, patients with prior mantle field radiotherapy who show signs of RIM should be closely monitored and referred to rehabilitation as early as possible.

\section{Statement of Ethics}

We confirm that we have read the Journal's position on issues involved in ethical publication and affirm that this report is consistent with those guidelines. Written informed consent was obtained from the patient and her son for publication of this case report and any accompanying images.

\section{Karger'}




\section{Conflict of Interest Statement}

None of the authors has any conflict of interest to disclose.

\section{Funding Sources}

No funding.

\section{Author Contributions}

J.A. planned and directed the study in data acquisition and interpretation. J.A., Y.E. and T.H. cared for this patient. Y.U., H.Y., M.A., N.H. and N.K. were involved in drafting the manuscript. M.T. and D.T. performed muscle pathology. All authors read and approved the manuscript.

\section{References}

1 Ng AK. Current survivorship recommendations for patients with Hodgkin lymphoma: focus on late effects. Blood. 2014;124(23):3373-9.

2 Ghosh PS, Milone M. Clinical and laboratory findings of 21 patients with radiation-induced myopathy. J Neurol Neurosurg Psychiatry. 2015;86(2):152-8.

3 van Leeuwen-Segarceanu EM, Dorresteijn LD, Pillen S, Biesma DH, Vogels OJ, van Alfen N. Progressive muscle atrophy and weakness after treatment by mantle field radiotherapy in Hodgkin lymphoma survivors. Int J Radiat Oncol Biol Phys. 2012;82(2):612-8.

4 Rowin J, Cheng G, Lewis SL, Meriggioli MN. Late appearance of dropped head syndrome after radiotherapy for Hodgkin's disease. Muscle Nerve. 2006;34(5):666-9.

5 Seidel C, Kuhnt T, Kortmann RD, Hering K. Radiation-induced camptocormia and dropped head syndrome: Review and case report of radiation-induced movement disorders. Strahlenther Onkol. 2015;191(10):76570 .

6 Furby A, Béhin A, Lefaucheur JP, Beauvais K, Marcorelles P, Mussini JM, et al. Late-onset cervicoscapular muscle atrophy and weakness after radiotherapy for Hodgkin disease: a case series. J Neurol Neurosurg Psychiatry. 2010;81(1):101-4. 